\title{
El aprendizaje móvil (m-learning) como herramienta formativa para la empresa
}

Mobile learning (m-learning) as training tool for the company

\author{
William-Oswaldo Aparicio-Gómez ${ }^{1}$ \\ Carlos-Alfonso Aparicio-Gómez ${ }^{2}$ \\ Ed\&TIC \\ Jenny Fabiola Hernández Niño ${ }^{3}$ \\ Politécnico Grancolombiano
}

Recibido: 01.11.2020

Aceptado: 15.11.2020

\section{Resumen}

El proyecto se enmarca en la línea de investigación denominada Proyectos educativos con el uso de las TIC, cuyo objetivo general es analizar las competencias digitales adquiridas a través de la formación m-learning; para lograrlo se plantearon los siguientes objetivos específicos: análisis de especificidad competencias digitales, análisis de especificidad formación mlearning, análisis y discusión sobre el nexo competencias digitales y formación m-learning en el campo empresarial.

\footnotetext{
1 waparicio@editic.net https://orcid.org/0000-0002-8178-1253

https://scholar.google.com/citations?user=V5RCdbUAAAAJ\&hl=es\&oi=ao

2 caparicio@editic.net https://orcid.org/0000-0002-3231-3699

https://scholar.google.com/citations?hl=es\&user=DCw7rvsAAAAJ

3 jfhernandez@poligran.edu.co https://orcid.org/0000-0003-0497-5422

https://scholar.google.com/citations?hl=es\&user=sAkOJa4AAAAJ
} 
Palabras clave: Educación, Aprendizaje móvil, Empresa, Competencias digitales.

\begin{abstract}
The project is part of the line of research called Educational projects with the use of ICT, whose general objective is to analyze the digital skills acquired through m-learning training; To achieve this, the following specific objectives were proposed: analysis of specificity of digital skills, analysis of specificity of m-learning training, analysis and discussion of the nexus of digital skills and m-learning training in the business field.
\end{abstract}

Keywords: Education, Mobile learning, Business, Digital skills.

\title{
1. Método
}

Se llevó a cabo una revisión bibliográfica sobre competencias digitales adquiridas a través de la formación m-learning. En primer lugar, se seleccionaron 30 artículos con especificidad en competencias digitales entre los años 2017 y 2020, y después de pasar diferentes filtros se escogen solamente 12 artículos que tienen incluidos temas relacionados con empresa tales como: negocios, gestión, contabilidad, ciencias de la decisión, entre otros. De la misma manera, y con criterios similares, se seleccionaron en segunda instancia 664 artículos con especificidad en formación M-learning entre los años 2017 y 2020, y después de pasar

diferentes filtros se seleccionan solamente 58 artículos que tratan sobre temas empresariales tales como: Negocios, gestión, contabilidad, y Ciencias de la decisión, entre otros. Por último, se hace el análisis y la discusión sobre el nexo: competencias digitales y formación m-learning. 


\section{Resultados}

\section{Especificidad competencias digitales 2017-2020: 30 artículos}

2020: 1 artículo

2019: 14 artículos

2018: 9 artículos

2017: 6 artículos

\section{Area temática:}

Negocios, gestión, contabilidad: 6 artículos

Ciencias de la decisión: 6 artículos

Ciencias de la computación: 5 artículos

Ciencias agropecuarias y biológicas: 1 artículo

Se seleccionan solamente las que tienen que ver aparentemente con temas relacionados con empresa:

Negocios, gestión, contabilidad: 6 artículos

Ciencias de la decisión: 6 artículos

En total: 12 artículos

Al hacer la trazabilidad, son exactamente los mismos artículos en las 2 áreas temáticas, con lo cual solo quedan 6 artículos para el análisis.

Con el fin de ampliar la bibliografía, se procede a hacer una lectura de los artículos de las otras áreas temáticas, encontrando que 6 pueden incluirse en el análisis que se está realizando: 5 artículos de ciencias sociales y uno (1) de Ciencias agropecuarias y biológicas. En total resultan 12 artículos para el análisis. 


\section{Especificidad formación m-learning 2017-2020: 664 artículos}

\section{Area temática:}

Ciencias de la Computación: 440 artículos

Ciencias Sociales: 291 artículos

Ingeniería: 185 artículos

Matemáticas: 60 artículos

Ciencias de decisión: 41 artículos

Negocios, Gestión y Contabilidad: 33 artículos

Artes y Humanidades: 31 artículos

Medicamentos: 31 artículos

Física y astronomía: 26 artículos

Energía: 20 artículos

Se seleccionan solamente los artículos que tratan sobre temas empresariales:

Negocios, gestión, contabilidad: 33 artículos

Ciencias de la decisión: 41 artículos

En total: 74 artículos

Al filtrar, resultan duplicados 16 artículos, resultando $\mathbf{5 8}$ artículos que tratan de temas como negocios, gestión, contabilidad y ciencias de la decisión.

En total, de las dos especificidades resultan para el análisis $12+58=\mathbf{7 0}$ artículos

\subsection{Análisis de especificidad sobre competencias digitales}

Doce (12) artículos para el análisis:

Artículo 1: La formación en competencias digitales de los profesionales de la salud en el lugar de trabajo] (Luque, 2019) 
Se concluye que las competencias digitales deben verse como competencias transversales en el diseño curricular de los profesionales de la salud. El dominio de tales competencias permitirá a los profesionales trabajar de manera eficiente en el contexto de la salud electrónica y la sociedad del conocimiento que se incluyeron en la capacitación para fomentar el "aprender haciendo

Palabras clave empleadas: Educación basada en competencias, Educación continua, competencias digitales, literatura saludable, alfabetización informacional, conocimiento administrativo, aprendizaje basado en problemas

\section{Artículo 2: Estudio comparativo de Competencias 2.0 entre jóvenes y adultos} mayores. Tiempo actual y desafíos para su inclusión (Artículo)(González García et al., 2019)

Esta investigación compara las habilidades digitales en conocimiento y uso de las TIC en comunicación social y aprendizaje colaborativo, habilidades útiles para buscar y tratar información, así como la competencia interpersonal entre estudiantes jóvenes y mayores de la Universidad de Granada (España).

Palabras clave empleadas: Competencias Digitales, Alfabetización digital, TIC, Jóvenes, Personas Mayores, Tecnologías de la Información y la Comunicación, Gente joven

Artículo 3: Discernimiento de los docentes por género en el uso de las TIC en el aula a partir de las competencias digitales] (Araiza \& Pedraza, 2019)

El estudio muestra las competencias digitales y el uso de las TIC, enfatizando la diferencia de aptitudes entre maestros y maestras.

Palabras clave empleadas: Habilidades digitales, Educación más alta, Tecnología de la información y comunicación, Formación del profesorado. 
Artículo 4: De los medios a las aulas. Actitudes de los futuros profesores de secundaria hacia la libertad de expresión y la participación en entornos digitales (Santiago-Del Pino et al., 2019)

El estudio trata sobre la opinión de futuros docentes de Secundaria, acerca de la libertad de expresión y la participación mediática.

Palabras clave empleadas: Discurso de odio, Educación mediática, Formación inicial de profesorado, Free speech Hate speech, Libertad de expresión, Media education, Media literacy, Pre-service teachers

Artículo 5: Economía digital en educación: Perspectivas y desarrollo. (Maymina et al., 2018)

El estudio demostró la necesidad de reformar el sistema educativo ruso mediante su transferencia a las tecnologías digitales para formar las competencias de los graduados en la demanda en el mercado laboral moderno.

Palabras clave empleadas: Conceptos de capacitación sobre innovaciones, Economía digital, Tecnologías educativas digitales, Economía digital, Conceptos de formación en innovaciones, Mercado de trabajo, Tecnologías educativas digitales.

Artículo 6: Estrategia de competitividad profesional basada en la apropiación de herramientas digitales. Caso Corporación Universitaria Americana, sede Medellín Colombia(Torres Taborda, 2018)

Se realiza una investigación sobre los Retos profesionales en un entorno cambiante, tomando como base la siguiente pregunta problematizadora: ¿Es sustentable la premisa de que las habilidades y competencias profesionales en el uso de herramientas TIC contribuyen al fortalecimiento del tejido empresarial colombiano? 
Palabras clave empleadas: Gestión del conocimiento, Information society, Information technology, Knowledge management, Productividad laboral, Responsabilidad social, Sociedad de la información, TIC.

Artículo 7: Perspectivas actuales de los docentes de Educación Básica y Medios acerca de la aplicación de las Competencias Tecnológicas en el aula (Hernández Suárez et al., 2018)

Este trabajo da cuenta de una investigación cuyo propósito ha sido buscar información acerca del modo en que los docentes de Educación Básica y Medios perciben su adaptación de las TIC a su trabajo docente para ajustarse a las nuevas realidades y expectativas de las sociedades de nativos digitales.

Palabras clave empleadas: Competencia tecnológica, Nativo digital, TIC, Nativo digital, Habilidad tecnológica.

Artículo 8: Una propuesta didáctica diseñada para favorecer el aprendizaje de la Inducción Electromagnética básica y el desarrollo de competencias digitales (Bravo et al., 2019)

Este artículo presenta y describe una propuesta de enseñanza diseñada para favorecer el aprendizaje del fenómeno de la inducción electromagnética (IE), así como el desarrollo de habilidades digitales generales. La propuesta se implementó en un curso con estudiantes de 17-18 años y los resultados obtenidos permiten percibir que la secuencia ha contribuido a lograr una mejor comprensión de las ideas clave asociadas con la IE, al mismo tiempo implicadas para los estudiantes en el uso de diferentes recursos tecnológicos y con ello el desarrollo de habilidades inherentes a dicho uso.

Palabras clave empleadas: Propuesta didáctica, Inducción electromagnética, TIC, Física, Enseñando. 


\section{Artículo 9: Desarrollo de competencias digitales en Comunidades virtuales: Un análisis} de "scolartic"(Martínez-Bravo et al., 2018)

La investigación analiza el desarrollo de habilidades digitales en la comunidad virtual ScolarTIC a través de un análisis de red y una encuesta entre sus usuarios. El estudio revela que en una estructura dinámica y descentralizada de intercambio de conocimientos puede ayudar a desarrollar diversas áreas de competencia digital. En el caso de ScolarTIC, las áreas de competencia digital más desarrolladas entre los usuarios son la seguridad y la información (cómo navegar, buscar, evaluar, almacenar y recuperar información, tanto en teléfonos inteligentes y tabletas como en otros dispositivos).

Palabras clave empleadas: Aprendizaje colaborativo, Alfabetización digital, Habilidad digital, Las TIC, Internet, Comunidades en línea.

Artículo 10: Competencias digitales clave de los Profesionales sanitarios (Montero Delgado et al., 2019)

El artículo presenta una serie de habilidades digitales para diferentes trabajadores de la salud con el fin de facilitar esta transición y mejorar su desempeño profesional con la ayuda de Internet y las tecnologías asociadas.

Palabras clave empleadas: Salud digital, Alfabetización digital en salud, Habilidades digitales, Transformación digital, La salud.

Artículo 11: El aprendizaje de los jóvenes con medios digitales fuera de la escuela: de lo informal a lo formal (Pereira et al., 2019)

Los principales resultados de la investigación confirman la existencia de una brecha entre la educación formal e informal. La educación informal está motivada principalmente por sus necesidades y la influencia de sus compañeros. Los colegas y la familia, junto con Internet y el autodescubrimiento, aparecen como fuentes importantes de conocimiento. 
Palabras clave empleadas: Aprendizaje informal, Competencias, Medios digitales, Educación en medios, Escuela, Aprendizaje informal, Investigación cualitativa, Jóvenes, La alfabetización mediática, Medios digitales, Investigación cualitativa, Colegio, Habilidades, Transmedia, Gente joven.

\section{Artículo 12: Evaluación de las competencias básicas de los veterinarios: implicaciones para las decisiones políticas en India (Artículo) (Sasidhar \& Suvedi, 2018)}

Los objetivos de este estudio fueron dobles: evaluar las competencias técnicas de los veterinarios y su aplicación, e investigar las actividades de los veterinarios y las formas apropiadas para que adquieran y mejoren sus competencias. El estudio se realizó en tres estados del sur de la India: Andhra Pradesh, Karnataka y Telangana.

Palabras clave empleadas: Competencia central, India, Veterinario.

\section{Palabras clave empleadas por los autores:}

Showing documents with keyword "aprendizaje informal", "collaborative learning", "competence-based education", "competencia tecnológica", "competencias", "competencias digitales", "conceptos de capacitación sobre innovaciones", "continuing education", "core competency", "didactic proposal", "digital competences", "digital economy", "digital educational technologies", "digital health", "digital health literacy", "digital literacy", "digital media", "digital native", "digital skill", "digital skills", "digital transformation", "discurso de odio", "economía digital", "educación en medios", "educación mediática", "ehealth", "electromagnetic induction", "escuela", "formación inicial de profesorado", "free speech", "gestión del conocimiento", "hate speech", "health literacy", "higher education", "ict", "icts", "india", "informal learning", "information literacy", "information society", "information technology", "information technology and communication", "innovations training concepts", "internet", "investigación cualitativa", "jóvenes", "knowledge management", "labor market", "labour productivity", "libertad de expresión", "media education", "media literacy", "medios digitales", "mercado de trabajo", "nativo digital", "online communities", "personas mayores", "physics", "pre-service teachers", "problem-based learning", "productividad laboral", 
"qualitative research", "responsabilidad social", "school", "seniors", "skills", "social responsibility", "sociedad de la información", "teacher training", "teaching", "technological skill", "tecnologías de la información y la comunicación", "tecnologías educativas digitales", "tic", "transmedia", "veterinarian", "young people". Search in All Documents.

\section{Palabras clave más empleadas por los autores:}

Innovation: 3 autores(Belle, 2019; Fragou et al., 2017a; Tamilarasan et al., 2019),

E-learning: 5 autores(Azeez \& Van Der Vyver, 2019; Bakhouyi et al., 2019; Balikaeva et al., 2018; Mphatsi, 2017; Snopce \& Fetaji, 2019)

M-Learning: todos los autores

Mobile Learning: todos los autores

Technology acceptance model: 3 autores(Al-Nawayseh et al., 2019a; Fabito, 2017; Fatima et al., 2017a).

\subsection{Análisis de especificidad sobre formación m-learning}

\section{Veintiún (21) artículos para el análisis:}

Se analizaron 58 artículos, de los cuales no se hace resumen de 37 por tener que ver solo con educación, aunque en el área temática de Scopus aparece como negocios, gestión, contabilidad o ciencias de la decisión:(Abdullah et al., 2019; Al-Arabiat et al., 2018; Al-Azawi \& Shakkah, 2018; Al-Nawayseh et al., 2019a; Albelali \& Alaulamie, 2019; Alsowayegh \& Garba, 2019; Azeez \& Van Der Vyver, 2019; Balikaeva et al., 2018; Bano et al., 2017; Belle, 2019; Bharati \& Srikanth, 2018; Deshpande \& Mangalwede, 2019; Dhanapal et al., 2019; Fabito, 2017; Farooq et al., 2019; Giannakas et al., 2019; Hassan et al., 2019; Imane et al., 2018; Jamaan \& Abdullah, 2018; Kirubakaran et al., 2018; Machado et al., 2017; Martins \& Gouveia, 2019a, 2019b; Melles et al., 2019; Metafas \& Politi, 2017; Mphatsi, 2017; Phuong Thao et al., 2019; Reddy et al., 2018; Remch et al., 2019; Sanchez-Azqueta et al., 2018; Sarrab et al., 2017, 2018; Shuja et al., 2019; Sri Istiyowati \& Prati, 2019; Srivastava et al., 2018; Syafar et al., 2019; Wainaina et al., 2019). 
De esta manera resultan solo 21 artículos que tratan de temas relacionados con la empresa, o con la forma en que la escuela les dará herramientas a sus estudiantes para desempeñarse luego en su actividad laboral a través de las M-Learning:

Artículo 1: Aplicaciones interactivas de aprendizaje Dzongkha para niños (Dolkar et al., 2018)

El objetivo de este documento es discutir sobre el desarrollo de una aplicación móvil para hacer que el aprendizaje de Dzongkha sea interesante.

Palabras clave empleadas: Aplicación Dzongkha; Dzongkha e-learning; Dzongkha mlearning; Aplicación para niños.

Artículo 2: Hacia un modelo conceptual para examinar el impacto de los factores de gestión del conocimiento en la aceptación del aprendizaje móvil. (Artículo) (Al-Emran et al., 2020)

El objetivo central del estudio es desarrollar un modelo conceptual al extender el modelo de aceptación de tecnología (TAM) con factores KM (adquisición, intercambio, aplicación y protección) para examinar la aceptación de M-learning. Los resultados desencadenaron que la adquisición, aplicación y protección del conocimiento tienen impactos positivos en la facilidad de uso y la utilidad percibidas.

Palabras clave empleadas: Conocimiento, administración, factores METRO-aprendizaje, Malasia, PLS-SEMTAM.

Artículo 3: Adopción del aprendizaje móvil en Jordania: factores que influyen en la tecnología. (Al-Nawayseh et al., 2019b) 
Se trata de un estudio cuantitativo que examina los factores que afectan la intención de los estudiantes de adoptar el m-learning utilizando la teoría unificada de aceptación y uso de la tecnología (UTAUT).

Palabras clave empleadas: Estoy aprendiendo; Modelo de aceptación de Tecnología; Modelo UTAUT.

Artículo 4: Hacia una arquitectura adaptativa para la integración de aplicaciones móviles con LMS utilizando la próxima generación de SCORM (Bakhouyi et al., 2019)

El artículo tiene como objetivo diseñar una arquitectura de software que se centre en particular en la integración de las especificaciones de próxima generación de SCORM para adaptar las características y mecanismos del sistema de gestión de aprendizaje y su extensión al escenario móvil.

Palabras clave empleadas: E-learning; Interoperabilidad; LMS; LRS; Estoy aprendiendo; Moodle SCORM; SOA; xAPI.

Artículo 5: El papel de la innovación y la autoeficacia en el turismo de aprendizaje virtual (Fatima et al., 2017b)

El estudio tiene como objetivo explorar la oportunidad que ofrece el aprendizaje móvil (mlearning) para la educación turística en el contexto de los países en desarrollo.

Palabras clave empleadas: Innovación; Aprendizaje móvil; Autoeficacia; Modelado de ecuaciones estructurales; Modelo de aceptación de Tecnología; Educación turística.

Artículo 6: Un proceso de diseño instruccional para crear una ecología U-learning. (Documento de sesión) (Fragou et al., 2017b) 
El artículo presenta los fundamentos, los problemas importantes y la metodología construidos en el contexto de UbiComp para definir inicialmente un proceso de diseño instruccional para construir una U - Learning Ecology para la educación multidisciplinaria.

Palabras clave empleadas: Innovación; Diseño instruccional; Internet de las Cosas; Ecología de aprendizaje; U-learning.

Artículo 7: Dando forma a los futuros sistemas de aprendizaje electrónico a través de las redes sociales dentro del contexto socio-técnico. (Documento de sesión) (Georgescu \& Bogoslov, 2018)

La investigación consistió en identificar el marco de factores críticos de éxito para los sistemas de aprendizaje electrónico y enfatizar cómo o por qué las redes sociales podrían considerarse el elemento clave que cumple con los requisitos de éxito para los futuros sistemas de elearning / m-learning, desde una perspectiva socio-técnica.

Palabras clave empleadas: E-Learning Perspectives; E-Learning Success Factors; Social Media; Socio-Technical Dimensions; Web-Based Education.

Artículo 8: Priorizar los requisitos de la aplicación de aprendizaje móvil (Khan et al., 2019)

La investigación se centró en el desarrollo de aplicaciones de aprendizaje móvil y su afectación debido a los cambios en la tecnología y los requisitos de los usuarios.

Palabras clave empleadas: Escenario de priorización de requisitos de M-learning; Requisitos de M-learning; Requisitos de aplicaciones móviles sce priorización; Aprendizaje móvil; Aplicación de aprendizaje móvil; Priorización de requisitos; Escenario de priorización de requisitos. 
Artículo 9: Uso de Learning Analytics para mejorar la eficacia de las herramientas de autoría móvil. (Krouska et al., 2019)

Los resultados de la investigación mostraron que la incorporación de Learning Analytics se considera realmente útil para los tutores que los apoyan a través de herramientas de autoría eficientes en entornos de m-learning.

Palabras clave empleadas: Herramienta de creación de contenido; Informes dinámicos; Análisis de aprendizaje; Aprendizaje móvil; Estrategias de enseñanza.

Artículo 10: Un modelo de aceptación de tecnologías móviles para la transferencia de conocimiento por parte de los empleados. (Kuciapski, 2017)

El propósito del estudio consistió en proponer un modelo conceptual basado en la teoría unificada de aceptación y uso de tecnología (UTAUT) para explicar los determinantes que afectan la intención de los empleados de usar dispositivos móviles y software para la transferencia de conocimiento durante el proceso de gestión del conocimiento.

Palabras clave empleadas: Conocimiento administrativo; El intercambio de conocimientos; Transferencia de conocimiento; Estoy aprendiendo; Aceptación de la tecnología; UTAUT.

Artículo 11: La adopción de una política de m-learning en la educación superior: la perspectiva de los profesionales en los países en desarrollo (Muhideen et al., 2019)

Los resultados obtenidos de la investigación indican que el uso de Thuto está aumentando constantemente, aunque la falta de tarifas de Internet altamente subsidiadas, acceso gratuito a Wi-Fi y pocas computadoras de laboratorio NUL que afectan negativamente el uso de Thuto en todo su potencial.

Palabras clave empleadas: Higher education; M-learning; Mobile learning; Policy innovation.

Artículo 12: Facilitar la participación grupal de los alumnos mediante agentes inteligentes en el M-learning colaborativo. (Njenga et al., 2018) 
Los hallazgos de la investigación mostraron niveles mejorados de construcción de conocimiento grupal en los grupos de tratamiento en comparación con el grupo control.

Palabras clave empleadas: Construcción de conocimiento grupal; Participación grupal; Agentes inteligentes; Estoy aprendiendo.

Artículo 13: Las utilidades de teorías de aprendizaje destacadas para el aprendizaje colaborativo móvil (MCL) con referencia a WhatsApp y M-learning. (Nyembe \& Howard, 2019)

El estudio encontró que algunas de las teorías de aprendizaje prominentes tienen mejores utilidades para MCL que otras, aunque todas tienen utilidades para MCL. En particular, las teorías de aprendizaje del aprendizaje colaborativo, las comunidades de práctica, el conectivismo, la teoría de la conversación y la teoría del aprendizaje social parecen tener utilidades directamente relacionadas para MCL y MCL en WhatsApp.

Palabras clave empleadas: Learning theories; Mobile collaborative learning (MCL); Mobile learning (M-learning); WhatsApp.

Artículo 14: Evaluación del patrón de diseño de software en el desarrollo de servicios basados en aplicaciones móviles relacionadas con el valor de mantenibilidad y modularidad (Panca et al., 2017)

El artículo presenta una implementación del patrón de diseño de software en el desarrollo de servicios basados en aplicaciones móviles. El objetivo principal de la investigación fue encontrar y evaluar la combinación de patrones de diseño de software relacionados con el valor de mantenibilidad y modularidad.

Palabras clave empleadas: Androide; Patrón de diseño; Mantenibilidad; Desarrollo de aplicaciones móviles; Modularidad; Métricas de calidad; SOA. 
Artículo 15: M-Learning the Future of Learning, análisis de sus posibilidades de uso (Snopce \& Fetaji, 2019)

El estudio de investigación analizó todos los factores más importantes que influyen en el mlearning.

Palabras clave empleadas: Desafíos educativos; comunicación y colaboración Introducción; e-learning aprendizaje móvil.

Artículo 16: La aplicación de aprendizaje de Byju: un estudio de investigación sobre la transformación del aprendizaje tradicional al aprendizaje personalizado basado en la tecnología (Sruthi \& Mukherjee, 2020)

El documento demuestra cómo la aplicación de Byju facilita y mejora la experiencia de enseñanza-aprendizaje entre los estudiantes de Kerala.

Palabras clave empleadas: Constructivismo; Aplicaciones de aprendizaje; Estoy aprendiendo; Plataformas de aprendizaje en línea; Aprendizaje personalizado; Tecnología; Aprendizaje Tradicional.

Artículo 17: Desafíos y capacidades de aprendizaje móvil. (Tamilarasan et al., 2019)

El artículo muestra cómo el M-learning no intenta suplantar la adaptación convencional, sino que la capacita para utilizar nuevas innovaciones y mejorar.

Palabras clave empleadas: Ambientes; Innovación; Pedagógico; Populacho; Psicológico; Impedimentos técnicos. 
Artículo 18: Comprender la aceptación y el uso de las aplicaciones de m-learning por parte de los empresarios: una aplicación de las teorías socio-cognitivas y motivacionales (Verkijika, 2019)

El estudio fue diseñado para examinar los factores que influyen en la aceptación de las aplicaciones de m-learning por parte de los empresarios.

Palabras clave empleadas: Emprendedores; Motivación extrínseca; Motivación intrínseca; Estoy aprendiendo; Teoría cognitiva social.

Artículo 19: Aprendizaje adaptado para optimizar las estrategias de tratamiento individualizado utilizando registros de salud electrónicos (Wu et al., 2020)

A través de estudios de simulación la investigación muestra que el aprendizaje M supera a los métodos existentes, aplican el M-learning para estimar los tratamientos óptimos personalizados de segunda línea para pacientes con diabetes tipo 2.

Palabras clave empleadas: Reglas de tratamiento individualizado; Pareo; Estudios observacionales; Medicina personalizada; Medicina de precisión.

Artículo 20: Preparación del aprendizaje móvil entre adultos que trabajan en Malasia (Yin Ling et al., 2018)

La investigación fue diseñada para determinar la preparación de los adultos que trabajan en el uso del aprendizaje móvil.

Palabras clave empleadas: aprendizaje móvil; preparación; adultos que trabajan.

Artículo 21: Los preliminares para las capacidades de los recursos humanos en las islas natuna: marco conceptual del eje marítimo mundial (Ying et al., 2017) 
El estudio diseñó una aplicación de aprendizaje móvil que promovió al gobierno regional de Natuna para preparar la mano de obra que se comunica en chino.

Palabras clave empleadas: Eje marítimo mundial; Estoy aprendiendo; Idioma mandarín; Natuna Turismo náutico.

\section{Palabras clave empleadas por los autores:}

Showing documents with keyword "adaptation", "adaptive learning", "ahp", "analytic hierarchy process", "andragogical factors", "android", "arcs", "augmented reality", "business and professional communication skills", "byju's", "cf", "class project assistant", "classroom inverted", "cloud computing (saas)", "clustering", "collaborative action research", "collaborative filtering", "collaborative research centre", "communication", "communication and collaboration introduction", "content authoring tool", "context", "continuance intention", "critical success factors", "deaf student", "design pattern", "dual-personae evaluators", "dynamic reporting", "dzongkha app", "dzongkha e-learning", "dzongkha m-learning", "e learning", "e- readiness", "e-learning", "e-learning perspectives", "e-learning success factors", "economic", "education", "educational application", "educational challenges", "educational technology", "efficiency", "elderly learners", "emerging issues", "english", "enjoyment", "enseñanza instruccional y aprendizaje personalizado", "entrepreneurs", "environments", "expectation confirmation model (ecm)", "extrinsic motivation", "facilitation discourse", "flexibility", "future specialists professional mobility", "gender difference", "global maritime axis", "group knowledge construction", "group organization", "group participation", "highschool students", "higher education", "higher education institutions", "ict", "ict competency", "ict-based learning", "inclusive pedagogy", "independent learning", "index terms: constructivism", "individualized treatment rules", "information success model", "information system success model (iss)", "innovation", "innovativeness", "instructional design", "instructional teaching and personalizad learning", "intelligent agents", "internet of things", "interoperability", "intrinsic motivation", "inverted classroom", "it-technologies", "kids' app", "knowledge management", "knowledge management factors", "knowledge sharing", 
"knowledge transfer", "learning", "learning analytics", "learning apps", "learning ecology", "learning from failure", "learning management", "learning theories", "learning theory", "lms", "location-based", "lrs", "m learning", "m-learning", "m-learning barriers", "m-learning pedagogies", "m-learning requirement prioritisation scenario", "m-learning requirements", "maintainability", "malaysia", "mandarin language", "matching", "mathematics", "media richness theory", "meeting room 1", "methodological approaches", "mixed inspection and testing", "mixed reality", "mlearning", "mobile application development", "mobile application requirements prioritisation sce", "mobile augmented reality", "mobile collaborative learning (mcl)", "mobile computing devices", "mobile device", "mobile devices", "mobile learning", "mobile learning (m-learning)", "mobile learning application", "mobile learning environments", "mobile learning readiness", "mobile-assisted learning", "mobile-dgbl", "mobile-learning", "modularity", "moodle", "moroccan university", "motivation", "natuna", "nautical tourism", "observational studies", "online learning", "online learning platforms", "open distance learning", "pacific", "participatory heuristic evaluation", "pedagogical", "pedagogical model", "personalization", "personalized learning", "personalized medicine", "pls-sem", "policy innovation", "populace", "precision medicine", "prioritisation", "projectbased learning", "psychological", "qr codes", "quality metrics", "readiness", "regulatory framework", "requirements prioritisation", "requirements prioritisation scenario", "research activities", "saudi arabia", "scorm", "sdg4", "second language learning", "secondary school", "security and privacy education", "self-education organization", "self-efficacy", "sentiment analysis", "soa", "social", "social cognitive theory", "social media", "social networking sites", "socio-technical dimensions", "south africa", "structural equation modelling", "students", "students adoption", "students' academic performance", "suitability", "sustainability", "tam", "teaching and learning", "teaching strategies", "technical and vocational education and training (t", "technical impediments", "technology", "technology acceptance", "technology acceptance model", "technology acceptance model (tam)", "the chi square test", "theory of innovation diffusion.", "theory of planned behavior", "tourism education", "traditional learning", "transformational learning", "u-learning", "ubiquitous learning", "ubiquitous technology", "university", "usability evaluations", "uses", "utaut", "utaut model", "utaut2", 
"vark", "virtual reality", "vocational education", "web-based education", "whatsapp", "working adults", "xapi". Search in All Documents.

\subsection{Análisis y discusión sobre el nexo competencias digitales y formación m-learning}

Haciendo análisis de las dos especificidades podemos concluir: Acerca de competencias digitales y su relación con el mundo laboral solo 5 artículos $(\mathbf{1 , 2 , 6 , 1 0 , 1 2 )}$ tratan explícitamente del tema.

Ciencias de la salud: El dominio de tales competencias permitirá a los profesionales trabajar de manera eficiente en el contexto de la salud electrónica y la sociedad del conocimiento que se incluyeron en la capacitación para fomentar el "aprender haciendo". Se concluye que las competencias digitales deben verse como competencias transversales en el diseño curricular de los profesionales de la salud (Luque, 2019).

Comunicación social: necesidad de incluir a las personas mayores en la tecnología, ya que es importante crear contacto de aprendizaje y comunicación (González García et al., 2019).

Funcionamiento organizacional: que las habilidades y competencias profesionales en el uso de herramientas TIC contribuyen al fortalecimiento del tejido empresarial colombiano (Torres Taborda, 2018).

Sistemas de salud: habilidades digitales para diferentes trabajadores de la salud con el fin de mejorar su desempeño profesional con la ayuda de Internet y las tecnologías asociadas (Montero Delgado et al., 2019).

Actividades de los veterinarios: Los hallazgos sugirieron que la capacitación previa al servicio, en servicio y de inducción básica, y la asistencia a seminarios, talleres y seminarios web nacionales e internacionales, son formas apropiadas de adquirir competencias (Sasidhar \& Suvedi, 2018). 
Acerca de M-learning y su relación con el mundo laboral solo 12 de los artículos $(\mathbf{2}, \mathbf{5}, 7,8,10,14,15,17,18,19,20,21)$ tratan explícitamente del tema:

Gestión del conocimiento: la adquisición, aplicación y protección del conocimiento tienen impactos positivos en la facilidad de uso y la utilidad percibidas (Al-Emran et al., 2020).

Turismo: los institutos de educación turística deben centrarse en la autoeficacia de los estudiantes para desarrollar una actitud positiva y una intención de comportamiento hacia el m-learning al lanzar servicios de educación basados en dispositivos móviles. (Fatima et al., 2017b).

Redes sociales dentro del contexto socio-técnico: las redes sociales podrían considerarse el elemento clave que cumple con los requisitos de éxito para los futuros sistemas de e-learning / m-learning, desde una perspectiva socio-técnica (Georgescu \& Bogoslov, 2018).

Ámbito educativo, comercial y de salud: El desarrollo de aplicaciones de aprendizaje móvil ve afectado debido a los cambios en la tecnología y los requisitos de los usuarios. (Khan et al., 2019).

Transferencia de conocimiento por parte de los empleados: la usabilidad de la tecnología en comparación con otras soluciones y la autonomía del usuario en la selección y el uso de aplicaciones tienen el mayor impacto en la intención de los empleados de usar dispositivos móviles y software para la transferencia de conocimiento (Kuciapski, 2017).

Desarrollo de servicios basados en aplicaciones móviles: combinación de patrones de diseño de software relacionados con el valor de mantenibilidad y modularidad. (Panca et al., 2017).

Posibilidades de uso de M-Learning: El aumento de las ventas de dispositivos móviles, el rápido crecimiento del tráfico web móvil, el crecimiento de la adopción de dispositivos 
móviles en el lugar de trabajo, entre otros factores, han llevado al desarrollo del mundo móvil del m-learning (Snopce \& Fetaji, 2019).

Aprendizaje móvil en diversos ámbitos: Después de llegar a casa, un teléfono celular es su método más ideal para aprender. M-learning no intenta suplantar la adaptación convencional, sino que la capacita para utilizar nuevas innovaciones y mejorar (Tamilarasan et al., 2019).

M-learning y los empresarios: Los resultados mostraron que tanto los factores motivacionales intrínsecos como los tuvieron una influencia positiva directa en las intenciones conductuales de adoptar aplicaciones de aprendizaje virtual (Verkijika, 2019).

Salud: aplicación de M-learning para estimar los tratamientos óptimos personalizados de segunda línea para pacientes con diabetes (Wu et al., 2020).

Trabajadores: El resultado mostró suficiente conocimiento, perspectiva positiva y acogió con satisfacción la integración del aprendizaje móvil en la educación entre los participantes (Yin Ling et al., 2018).

Recursos humanos: El estudio aconsejó un aprendizaje móvil basado en lo que destaca la utilización de la infraestructura tecnológica y la aplicación de Internet en el aprendizaje (Ying et al., 2017) .

\section{Conclusiones}

Acerca de competencias digitales y su relación con el mundo laboral solo 5 artículos resultaron pertinentes para nuestro estudio con los siguientes temas: Ciencias de la salud, comunicación social, funcionamiento organizacional, Sistemas de salud, y Actividades de los veterinarios.

Acerca de M-learning y su relación con el mundo laboral solo 12 de los artículos tratan explícitamente del tema: Gestión del conocimiento, turismo, redes sociales dentro del 
contexto socio-técnico, ámbito educativo, comercial y de salud; transferencia de conocimiento por parte de los empleados, desarrollo de servicios basados en aplicaciones móviles, posibilidades de uso de M-Learning, aprendizaje móvil en diversos ámbitos, M-learning y los empresarios, Salud, trabajadores, y Recursos humanos.

\section{Referencias}

Abdullah, S. A., Saud, M. S., \& Kamin, Y. (2019). M-learning for technical and vocational education training (TVET). International Journal of Recent Technology and Engineering, 8(3), 7236-7239. https://doi.org/10.35940/ijrte.C6291.098319

Al-Arabiat, D. A., Ahmad, W. F. W., \& Sarlan, A. (2018, October 25). Mobile Learning and Software as a Service: The Effect on Knowledge Sharing. 2018 4th International Conference on Computer and Information Sciences: Revolutionising Digital Landscape for Sustainable Smart Society, $\quad$ ICCOINS $2018 \quad$ - $\quad$ Proceedings. https://doi.org/10.1109/ICCOINS.2018.8510616

Al-Azawi, R., \& Shakkah, M. S. (2018). Embedding augmented and virtual reality in educational learning method: Present and future. 2018 9th International Conference on Information and Communication Systems, ICICS 2018, 2018-Janua, 218-222. https://doi.org/10.1109/IACS.2018.8355470

Al-Emran, M., Mezhuyev, V., \& Kamaludin, A. (2020). Towards a conceptual model for examining the impact of knowledge management factors on mobile learning acceptance. Technology in Society, 61. https://doi.org/10.1016/j.techsoc.2020.101247

Al-Nawayseh, M. K., Baarah, A. H., Al-Masaeed, S. A., \& Alnabhan, M. M. (2019a). Mobile learning adoption in Jordan: Technology influencing factors. International Journal of $\begin{array}{llll}\text { Networking and } \quad \text { Virtual } & \text { Organisations, }\end{array}$ https://doi.org/10.1504/IJNVO.2019.100600 
Al-Nawayseh, M. K., Baarah, A. H., Al-Masaeed, S. A., \& Alnabhan, M. M. (2019b). Mobile learning adoption in Jordan: Technology influencing factors. International Journal of Networking and Virtual Organisations, 20(4), 400-417. https://doi.org/10.1504/IJNVO.2019.100600

Albelali, S. A., \& Alaulamie, A. A. (2019, May 1). Gender Differences in Students' Continuous Adoption of Mobile Learning in Saudi Higher Education. 2nd International Conference on Computer Applications and Information Security, ICCAIS 2019. https://doi.org/10.1109/CAIS.2019.8769554

Alsowayegh, N., \& Garba, I. (2019). WhatsApp for Defamiliarizing Foundation Year English Learners: A Collaborative Action Research Evaluation. Smart Innovation, Systems and Technologies, 150, 91-97. https://doi.org/10.1007/978-3-030-22964-1_10

Araiza, M. D. J., \& Pedraza, E. (2019). Discernment of teachers by gender in the use of ICT in the classroom based on digital competences. Espacios, 40(21).

Azeez, N. A., \& Van Der Vyver, C. (2019). Digital Education: Assessment of e-Learning and m-Learning Adoption in Tertiary Institutions in South Africa. 2018 IEEE Conference on ELearning, e-Management and e-Services, IC3e 2018, 23-28. https://doi.org/10.1109/IC3e.2018.8632654

Bakhouyi, A., Dehbi, R., \& Talea, M. (2019, May 1). Toward an Adaptive Architecture for Integrating Mobile Apps with LMS using Next Generation of SCORM. 2nd International Conference on Computer Applications and Information Security, ICCAIS 2019. https://doi.org/10.1109/CAIS.2019.8769575

Balikaeva, M. B., Litvak, R. A., Grevtseva, G. Y., \& Pavlichenko, A. A. (2018). Analysis of Students Self-education Forms Abroad for the Professional Mobility Formation Using ITtechnologies. Proceedings of the 2018 International Conference "'Quality Management, 
Transport and Information Security, Information Technologies"”, IT and QM and IS 2018, 775-778. https://doi.org/10.1109/ITMQIS.2018.8524930

Bano, M., Zowghi, D., \& Kearney, M. (2017). Feature based sentiment analysis for evaluating the mobile pedagogical affordances of apps. IFIP Advances in Information and Communication Technology, 515, 281-291. https://doi.org/10.1007/978-3-319-74310-3_30

Belle, L. J. (2019). An Evaluation of a Key Innovation: Mobile Learning. Academic Journal of Interdisciplinary Studies, 8(2), 39-45. https://doi.org/10.2478/ajis-2019-0014

Bharati, V. J., \& Srikanth, R. (2018). Modified UTAUT2 model for m-learning among students in India. International Journal of Learning and Change, 10(1), 5-20. https://doi.org/10.1504/IJLC.2018.089532

Bravo, B., Bouciguez, M. J., \& Braunmüller, M. (2019). A didactic proposal designed to favor the learning of the Electromagnetic Induction and the development of digital competences. $\begin{array}{lll}\text { Revista } & \text { Eureka, } & 16(1) .\end{array}$ https://doi.org/10.25267/Rev_Eureka_ensen_divulg_cienc.2019.v16.i1.1203

Deshpande, S. B., \& Mangalwede, S. R. (2019). The effect of contexts on learning styles in m-learning environment using chi square test. International Journal of Recent Technology and Engineering, 8(3), 359-364. https://doi.org/10.35940/ijrte.C4173.098319

Dhanapal, S., Salman, N. W., Raman Kutty, G. K., Ansari, K. M. S., \& Shah, S. A. (2019). "XYZ" application as a tool for teaching and learning in institutions of higher learning: An exploratory study. Jurnal Pengurusan, 55. https://doi.org/10.17576/pengurusan-2019-55-04

Dolkar, K., Tshering, S., Bidha, C., Thinley, D., \& Dhungyel, P. R. (2018). Interactive Dzongkha Learning Apps for Kids. Proceedings - 2017 International Conference on Information Technology, ICIT 2017, 268-271. https://doi.org/10.1109/ICIT.2017.28 
Fabito, B. S. (2017). Exploring critical success factors of mobile learning as perceived by students of the College of Computer Studies - National University. Proceedings - 2017 International Conference on Soft Computing, Intelligent System and Information Technology: Building Intelligence Through IOT and Big Data, ICSIIT 2017, 2018-Janua, 220-226. https://doi.org/10.1109/ICSIIT.2017.25

Farooq, E., Iqbal, S., \& Ghani, M. A. N. U. (2019, November 1). Constructive Alignment: Investigating Student's Use of Mobile Technologies in University Classrooms. 3rd International Conference on Innovative Computing, ICIC 2019. https://doi.org/10.1109/ICIC48496.2019.8966702

Fatima, J. K., Ghandforoush, P., Khan, M., \& Masico, R. Di. (2017a). Role of innovativeness and self-efficacy in tourism m-learning. Tourism Review, 72(3), 344-355. https://doi.org/10.1108/TR-02-2017-0019

Fatima, J. K., Ghandforoush, P., Khan, M., \& Masico, R. Di. (2017b). Role of innovativeness and self-efficacy in tourism m-learning. Tourism Review, 72(3), 344-355. https://doi.org/10.1108/TR-02-2017-0019

Fragou, O., Kameas, A., \& Zaharakis, I. D. (2017a). An instructional design process for creating a U-learning ecology. IEEE Global Engineering Education Conference, EDUCON, 1817-1823. https://doi.org/10.1109/EDUCON.2017.7943097

Fragou, O., Kameas, A., \& Zaharakis, I. D. (2017b). An instructional design process for creating a U-learning ecology. IEEE Global Engineering Education Conference, EDUCON, 1817-1823. https://doi.org/10.1109/EDUCON.2017.7943097

Georgescu, R. M., \& Bogoslov, I. A. (2018). Shaping future e-learning systems through social media within the socio-technical context. Proceedings of the 32nd International Business Information Management Association Conference, IBIMA 2018 - Vision 2020: Sustainable 
Economic Development and Application of Innovation Management from Regional Expansion to Global Growth, 8065-8075.

Giannakas, F., Papasalouros, A., Kambourakis, G., \& Gritzalis, S. (2019). A comprehensive cybersecurity learning platform for elementary education. Information Security Journal, 28(3), 81-106. https://doi.org/10.1080/19393555.2019.1657527

González García, E., Amaro Agudo, A., \& Martínez Heredia, N. (2019). Comparative study of Competences 2.0 between young and senior people. Present time and challenges for their inclusion. Espacios, 40(5).

Hassan, M. M., Tukiainen, M., \& Qureshi, A. N. (2019, December 1). Participatory Heuristic Evaluations of Jeliot Mobile : End-users evaluating usability of their mlearning application. TIMES-ICON 2019 - 2019 4th Technology Innovation Management and Engineering Science International Conference. https://doi.org/10.1109/TIMES-iCON47539.2019.9024452

Hernández Suárez, C. A., Prada Núñez, R., \& Ramirez Leal, P. (2018). Current perspectives of teachers of elementary and middle education about the application of the technological competences in the classroom. Espacios, 39(43).

Imane, E. H., Slimani, K., Otmane, O., Bourekkadi, S., Abdelouahab, I., \& Aziz, B. (2018). Moroccan university students' perspectives concerning english mobile learning. Proceedings of the 31st International Business Information Management Association Conference, IBIMA 2018: Innovation Management and Education Excellence through Vision 2020, 1163-1176.

Jamaan, J., \& Abdullah, Z. S. (2018). Assessing Arabic language learning for deaf student: A study of using mobile immersion in Saudi Arabia. Proceedings - International Conference on Information and Communication Technology for the Muslim World 2018, ICT4M 2018, 296300. https://doi.org/10.1109/ICT4M.2018.00061 
Khan, A. I., Al-Khanjari, Z., \& Sarrab, M. (2019). Prioritising mobile learning application requirements. International Journal of Business Information Systems, 32(1), 91-108. https://doi.org/10.1504/IJBIS.2019.102705

Kirubakaran, R., Francis Jency, X., \& Aswini, D. (2018). Mobile learning for education in India-A feasibility study. International Journal of Recent Technology and Engineering, 7(4), 386-389.

Krouska, A., Troussas, C., \& Virvou, M. (2019, July 1). Using Learning Analytics to Improve the Efficacy of Mobile Authoring Tools. 10th International Conference on Information, Intelligence, $\quad$ Systems and $\quad$ Applications, 2019. https://doi.org/10.1109/IISA.2019.8900726

Kuciapski, M. (2017). A model of mobile technologies acceptance for knowledge transfer by employees. Journal of Knowledge Management, 21(5), 1053-1076. https://doi.org/10.1108/JKM-03-2016-0136

Luque, A. M. F. (2019). On-the-job digital competence training for health professionals. Revista Cubana de Informacion En Ciencias de La Salud, 30(2).

Machado, L. R., da Silva Mendes, J. S., Sampaio, D. C. F., Grande, T. P. F., \& Behar, P. A. (2017). M-learning and the elderly: Construction of inclusive pedagogies. Smart Innovation, Systems and Technologies, 75, 391-399. https://doi.org/10.1007/978-3-319-59451-4_39

Martínez-Bravo, M. C., Sádaba Chalezquer, C., \& Serrano-Puche, J. (2018). Delevopment of digital competences in online communities: An Analysis of scolartic. Prisma Social, 20, 129159.

Martins, E. R., \& Gouveia, L. M. B. (2019a). ML-SAI: Pedagogical model based on inverted classroom intended for m-learning activities. Espacios, 40(36). 
Martins, E. R., \& Gouveia, L. M. B. (2019b). Pedagogical model ML-SAI: Reflections on methodological approaches. Espacios, 40(36).

Maymina, E., Divina, T., \& Liulia, V. (2018). Digital economy in education: Perspectives and development perspectives. Espacios, 39(38).

Melles, G., Winfree, T., \& Graham, P. (2019). Collaborative design of mobile interfaces for vocational building and construction education. Smart Innovation, Systems and Technologies, 134, 641-651. https://doi.org/10.1007/978-981-13-5974-3_56

Metafas, D., \& Politi, A. (2017). Mobile-assisted learning: Designing class project assistant, a research-based educational app for project based learning. IEEE Global Engineering $\begin{array}{lll}\text { Education } & \text { Conference, } & \text { 667-675CON, }\end{array}$ https://doi.org/10.1109/EDUCON.2017.7942918

Montero Delgado, J. A., Merino Alonso, F. J., Monte Boquet, E., Ávila de Tomás, J. F., \& Cepeda Díez, J. M. (2019). Key digital skills for healthcare professionals. Educacion Medica. https://doi.org/10.1016/j.edumed.2019.02.010

Mphatsi, L. (2017, November 8). Technology-enhanced learning through Sakai (Thuto) at the National University of Lesotho. 2017 IST-Africa Week Conference, IST-Africa 2017. https://doi.org/10.23919/ISTAFRICA.2017.8102314

Muhideen, S., Yen, Y., Iddrisu, S., Mohammed, M. A., \& Bisanda, B. W. (2019). The adoption of an m-learning policy in higher education: The professionals' perspective in developing countries. Humanities and Social Sciences Letters, 7(1), 29-45. https://doi.org/10.18488/journal.73.2019.71.29.45 
Njenga, S., Oboko, R., Omwenga, E., \& Maina, E. (2018, July 20). Facilitating group learner participation using intelligent agents in collaborative M-learning. 2018 IST-Africa Week Conference, IST-Africa 2018.

Nyembe, B. Z. M., \& Howard, G. R. (2019, August 1). The utilities of prominent learning theories for mobile collaborative learning (MCL) with reference to WhatsApp and Mlearning. IcABCD 2019 - 2nd International Conference on Advances in Big Data, Computing and Data Communication Systems. https://doi.org/10.1109/ICABCD.2019.8851042

Panca, B. S., Mardiyanto, S., \& Hendradjaya, B. (2017, May 30). Evaluation of software design pattern on mobile application based service development related to the value of maintainability and modularity. Proceedings of 2016 International Conference on Data and Software Engineering, ICoDSE 2016. https://doi.org/10.1109/ICODSE.2016.7936132

Pereira, S., Fillol, J., \& Moura, P. (2019). Young people learning from digital media outside of school: The informal meets the formal. Comunicar, 27(58), 41-50. https://doi.org/10.3916/C58-2019-04

Phuong Thao, T. T., Thai, L. D., Thanh, H. T., Tran, T., Tuyet Trinh, L. T., \& Vuong, Q. H. (2019). Mobile learning for high-school mathematics as a path to better sustainability in a fastchanging society: An exploratory study from Vietnam. Problems and Perspectives in Management, 17(2), 392-403. https://doi.org/10.21511/ppm.17(2).2019.30

Reddy, E., Sharma, B., Reddy, P., \& Dakuidreketi, M. (2018). Mobile Learning Readiness and ICT Competency: A Case Study of Senior Secondary School Students in the Pacific Islands. Proceedings - 2017 4th Asia-Pacific World Congress on Computer Science and Engineering, APWC $\quad$ on $\quad$ CSE 137-143. https://doi.org/10.1109/APWConCSE.2017.00031 
Remch, Z., Bourekkadi, S., Khoulji, S., Lechhab, A., Fakhri, Y., \& Kerkeb, M. L. (2019). An analysis approach on Mobile Learning and its impact on student's performance and comportments. Proceedings of the 33rd International Business Information Management Association Conference, IBIMA 2019: Education Excellence and Innovation Management through Vision 2020, 6370-6376.

Sanchez-Azqueta, C., Celma, S., Aldea, C., Gimeno, C., \& Cascarosa, E. (2018). Using hyperdata in a laboratory of electronics QR codes applied to experimental learning. IEEE Global Engineering Education Conference, EDUCON, 2018-April, 467-471. https://doi.org/10.1109/EDUCON.2018.8363267

Santiago-Del Pino, M., Romero-Oliva, M. F., \& Goenechea-Permisán, C. (2019). From media to classrooms. Attitudes of prospective teachers towards freedom of expression and participation in digital environments. Espacios, 40(2).

Sarrab, M., Al-Shihi, H., Al-Khanjari, Z., \& Bourdoucen, H. (2018). Development of mobile learning application based on consideration of human factors in Oman. Technology in Society, 55, 183-198. https://doi.org/10.1016/j.techsoc.2018.07.004

Sarrab, M., Al-Shihi, H., \& Al Shib, I. N. S. (2017). Mobile learning key influencing factors adoption based on analytic hierarchy process. International Journal of Information and Decision Sciences, 9(4), 387-404. https://doi.org/10.1504/IJIDS.2017.088109

Sasidhar, P. V. K., \& Suvedi, M. (2018). Assessment of the core competencies of veterinarians: implications for policy decisions in India. Revue Scientifique et Technique (International Office of Epizootics), 37(3), 785-795. https://doi.org/10.20506/37.3.2885

Shuja, A., Qureshi, I. A., Schaeffer, D. M., \& Zareen, M. (2019). Effect of m-learning on students' academic performance mediated by facilitation discourse and flexibility. Knowledge Management and E-Learning, 11(2), 158-200. https://doi.org/10.34105/j.kmel.2019.11.009 
Snopce, H., \& Fetaji, M. (2019, October 1). M-Learning the Future of Learning, Analysis of its Usage Possibilities. 3rd International Symposium on Multidisciplinary Studies and Innovative $\quad$ Technologies, $\quad$ ISMSIT $2019 \quad$ - $\quad$ Proceedings. https://doi.org/10.1109/ISMSIT.2019.8932726

Sri Istiyowati, L., \& Prati, P. (2019). Mobile learning readiness in higher eduction based on the theory of planned behavior. International Journal of Recent Technology and Engineering, 8(1C2), 831-836.

Srivastava, A., Verma, P., \& Tripathi, A. P. (2018). M-learning interventions for instructional teaching. Espacios, 39(49).

Sruthi, P., \& Mukherjee, S. (2020). Byju's the learning app: An investigative study on the transformation from traditional learning to technology based personalized learning. International Journal of Scientific and Technology Research, 9(3), 5054-5059.

Syafar, F., Sidik, D., \& Husain, H. (2019). Students' adoption of mobile learning: An investigation in Indonesia higher education. Proceedings of the 33rd International Business Information Management Association Conference, IBIMA 2019: Education Excellence and Innovation Management through Vision 2020, 7641-7647.

Tamilarasan, P., Karthick, S., \& Anupama, C. G. (2019). Mobile learning challenges and capabilities. International Journal of Recent Technology and Engineering, 8(3), 5358-5361. https://doi.org/10.35940/ijrte.C6883.098319

Torres Taborda, S. L. (2018). Professional competitiveness strategy based on the appropriation of digital tools. Case of the American University Corporation, Medellin, Colombia. Espacios, 39(50). 
Verkijika, S. F. (2019). Understanding the acceptance and use of m-learning apps by entrepreneurs: An application of the social-cognitive and motivational theories. Information Resources Management Journal, 32(4), 42-55. https://doi.org/10.4018/IRMJ.2019100103

Wainaina, P. K., Maina, E. M., \& Nzuki, D. M. (2019, May 1). A Review of Mobile Learning Considerations in Open Distance Learning and Access to Higher Education. 2019 IST-Africa Week Conference, IST-Africa 2019. https://doi.org/10.23919/ISTAFRICA.2019.8764821

Wu, P., Zeng, D., \& Wang, Y. (2020). Matched Learning for Optimizing Individualized Treatment Strategies Using Electronic Health Records. Journal of the American Statistical Association, 115(529), 380-392. https://doi.org/10.1080/01621459.2018.1549050

Yin Ling, A. M., Wan Ahmad, W. F., \& Hashim, A. S. (2018, October 25). Readiness of Mobile Learning among Working Adults in Malaysia. 2018 4th International Conference on Computer and Information Sciences: Revolutionising Digital Landscape for Sustainable Smart Society, $\quad$ ICCOINS $2018 \quad$ - $\quad$ Proceedings. https://doi.org/10.1109/ICCOINS.2018.8510590

Ying, Y., Mursitama, T. N., Oktriono, K., \& Abbas, B. S. (2017). The preliminaries for human resources' capabilities in natuna Islands: Global maritime axis conceptual framework. 2017 3rd International Conference on Information Management, ICIM 2017, 506-510. https://doi.org/10.1109/INFOMAN.2017.7950437 
REVISTA INTERNACIONAL DE PEDAGOGÍA E INNOVACIÓN EDUCATIVA |

Volumen 1. Número 1. Enero - Junio 2021

ISSN: 2745-0341 (En línea) 